\title{
INFLUENCE OF MAGNETIC FIELD ON BLUE ANTI-STOKES LUMINESCENCE OF ZnSe: Cr CRYSTALS
}

\author{
V.YU. IVANOV \\ Department of Physics, University of Dnepropetrovsk \\ 320625, Dnepropetrovsk, Ukraine
}

Yu.G. Semenov

Institute of Semiconductors Physics, National Acad. of Science, 252650, Kiev, Ukraine

M. SuRma AND M. Godlewski

Institute of Physics, Polish Academy of Sciences

Al. Lotników 42/36, 02-668 Warsaw, Poland

The results of photoluminescence and magneto-luminescence studies of chromium doped $\mathrm{ZnSe}$ crystals are presented for blue colour shallow donor-shallow acceptor pair photoluminescence, with a zero phonon line at $2.692 \mathrm{eV}$. This donor-acceptor pair photoluminescence is observed under photo-excitation with photon energies smaller than the emission energy (anti-Stokes luminescence) and dominates in the photoluminescence spectrum of $\mathrm{ZnSe}: \mathrm{Cr}$ at the excitation energy about $2.41 \mathrm{eV}$ and for temperature $T<20 \mathrm{~K}$. A quant um efficiency of this anti-Stokes luminescence is relatively large. It is of about $10^{-3}$ at $0 \mathrm{~T}$ and increases with increasing magnetic field up to $7 \mathrm{~T}$ at temperature $T=2 \mathrm{~K}$. We relate the intensity of the anti-Stokes luminescence to a non-equilibrium concentration of photo-excited $\mathrm{Cr}^{+}$ions and propose that the rate of spin-dependent recombination of $\mathrm{Cr}^{+}$ions with free holes (decreases with increasing magnetic field) is responsible for the observed increase in the anti-Stokes luminescence intensity at higher magnetic fields.

PACS numbers: $71.55 . \mathrm{Gs}, 78.55 . \mathrm{Et}, 76.30 . \mathrm{Fc}$

\section{Introduction}

We have reported recently the observation of the anti-Stokes luminescence (ASL) emission in chromium doped ZnSe crystals [1]. By the ASL emission we mean here photoluminescence (PL) emission observed at photon energies larger or much larger than excitation energy. A blue colour shallow donor-shallow acceptor pair (DAP) PL with zero phonon line at $2.692 \mathrm{eV}$ was observed under $2.41 \mathrm{eV}$ excitation. This ASL process has a relatively high quantum efficiency of about $10^{-3}$. 
We have tentatively explained the appearance of this ASL emission by a two-step excitation process involving two complementary ionization transitions of $\mathrm{Cr}$ ion in $\mathrm{ZnSe}$ [1]. In this communication we positively verify our model based on new results of the PL and magneto-luminescence investigations performed for a series of $\mathrm{ZnSe}: \mathrm{Cr}$ samples with different $\mathrm{Cr}$ concentrations.

\section{Experimental}

ZnSe crystals studied, grown from melt by Bridgman-Stockbarger method, were treated in zinc melt, to reduce concentration of zinc vacancies, and were purified by a zone melting. These samples were intentionally doped to the melt with chromium and chromium concentration varied between $10^{16}$ and $5 \times 10^{19} \mathrm{~cm}^{-3}$.

PL and magneto-luminescence measurements were performed on conventional setups described elsewhere [2].

\section{Results and discussion}

In Fig. 1 we present the PL spectrum of chromium doped ZnSe observed for two excitation energies: (a) for the photon energy close to the band-to-band transition of $\mathrm{ZnSe}$ and (b) for the photon energy within $2+$ to $1+$ ionization band of $\mathrm{Cr}$ in $\mathrm{ZnSe}$. The detailed analysis of the observed PL spectrum was given previously $[1,2]$. Here we concentrate our attention on the shallow DAP PL, with a zero phonon line at $2.692 \mathrm{eV}$, observed upon excitation with photon energies below the emission energy. We will further call this PL the ASL emission.

The excitation spectrum of the ASL emission was measured and was shown to be similar to the ionization absorption of $\mathrm{Cr}\left(\mathrm{Cr}^{2+}\right.$ to $\left.\mathrm{Cr}^{1+}\right)$, which was a basis of our model relating the ASL emission to the two-step ionization $(2+$ to $1+$, $1+$ to $2+$ ) transitions of $\mathrm{Cr}$ in $\mathrm{ZnSe}[1,2]$. IIoles (then partly trapped by shallow acceptors) are created in the valence band (VB) in the $\mathrm{Cr}^{2+} \rightarrow \mathrm{Cr}^{1+}+\mathrm{h}_{\mathrm{VB}}$ ionization transition. The photo-excited $\mathrm{Cr}^{1+}$ states can be metastably occupied at low temperatures, which allows for excitation of free electrons in the conduction band (CB) (then partly trapped by shallow donors) in the complementary $\mathrm{Cr}^{1+} \rightarrow$ $\mathrm{Cr}^{2+}+e_{\mathrm{CB}}$ ionization process.

Formally, for the two-step ionization mechanism of the ASL excitation, a quadratic dependence of the ASL intensity on excitation intensity should be observed. However, such dependence was only observed (Fig. 2) for relatively low excitation intensities. For increased excitation intensities a linear dependence was found. Such linear dependence can still be explained assuming the two-step excitation mechanism of the ASL excitation under assumptions that holes are predominantly trapped by shallow acceptors (and less efficiently by $\mathrm{Cr}^{1+}$ centers) or that $\mathrm{Cr}^{1+}$-acceptors tunneling processes are efficient in our samples [2]. IIowever, such linear dependence can also mean that $\mathrm{Cr}-\mathrm{DAP}$ co-operative transitions are efficient and are responsible for the ASL excitation. We have recently pointed out that one such process can be efficient in $\mathrm{ZnSe}: \mathrm{Cr}$ [2]. We mean here an Auger-type energy transfer process, in which recombination energy of $\mathrm{Cr}^{1+}+\mathrm{h}_{\mathrm{VB}} \rightarrow \mathrm{Cr}^{2+}$ capture process is transferred to a nearby deep acceptor of $\mathrm{ZnSe}$ and is used for its ionization. In such case, free holes are excited in the VB in the $2+$ to $1+$ ionization transition of $\mathrm{Cr}$ and free electrons in the $\mathrm{CB}$ in the ionization transition of 


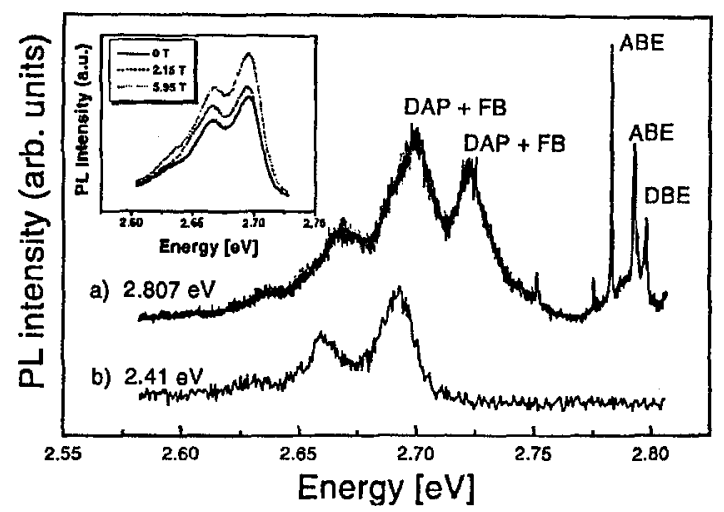

Fig. 1. Photoluminescence spectrum of $\mathrm{ZnSe}: \mathrm{Cr}$ crystal (chromium concentration $4 \times 10^{18} \mathrm{~cm}^{-3}$ ) measured at $4.2 \mathrm{~K}$ under two excitation energies: $2.807 \mathrm{eV}$ (a) and $2.41 \mathrm{eV}(\mathrm{b})$. In the inset we show PL emission for $2.41 \mathrm{eV}$ excitation energy and for external magnetic field equal to $0 \mathrm{~T}, 2.15 \mathrm{~T}$ and $5.95 \mathrm{~T}$. DBE (donor bound exciton), $\mathrm{ABE}$ (acceptor bound exciton), DAP and FB (free-to-bound) denote different recombination transitions observed.

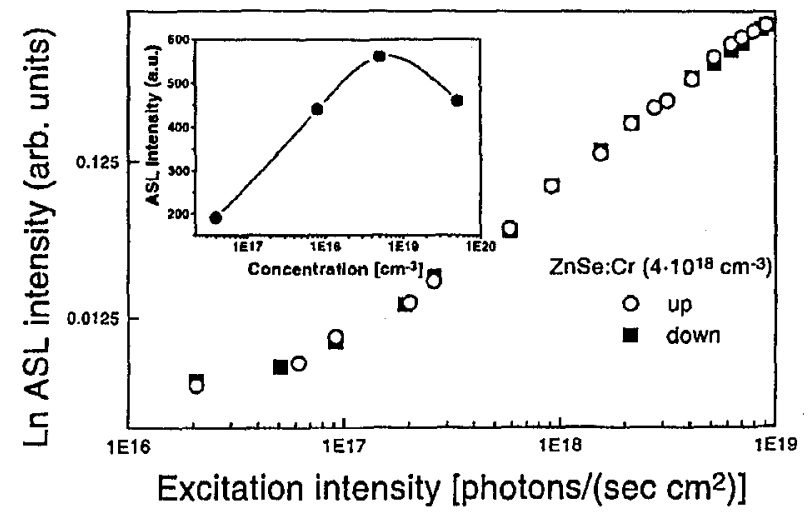

Fig. 2. Dependence of the ASL intensity on the intensity of excitation (for $2.41 \mathrm{eV}$ photon energy). Up and down stands for the results obtained with increasing (up) and decrrasing (down) light intensities. In the inset we show the dependence of the ASL intensity on chromium concentration in the sample.

a deep acceptor. This Auger-type ASL excitation process can be efficient only if retrapping of holes by $\mathrm{Cr}^{1+}$ ions is efficient.

The above mechanism of the ASL excitation may be efficient since for ZnSe $2+$ to $1+$ ionization transition of $\mathrm{Cr}$ partly overlaps with ionization transitions of two deep acceptors of $\mathrm{ZnSe}-\mathrm{Cu}$-related $0.4 \mathrm{eV}$ acceptor $[3,4]$ and zinc vacancy and Cu-related $0.7 \mathrm{eV}$ acceptor $[3,4]$. However, such an overlap of ionization energies opens a question if the ASL excitation is really Cr-related. To verify this point we have performed the $P L$, photo-conductivity and electron spin resonance 
(ESR) experiments on a series of $\mathrm{ZnSe}$ : $\mathrm{Cr}$ samples with a varying $\mathrm{Cr}$ concentration but with similar concentrations of lattice contaminants, such as e.g. copper. The results of the latter two experiments are not discussed here since they are fully consistent with those reported previously $[1,2]$.

The dependence of the ASL emission on $\mathrm{Cr}$ concentration is shown in the inset of Fig. 2. The ASL intensity increases with increasing $\mathrm{Cr}$ concentration in our samples up to relatively high concentrations. Then a decrease in the intensity of the ASL and of green and red deep DAP PLs is observed, which is consistent with an increased efficiency of nonradiative recombination processes in heavily $\mathrm{Cr}$ doped samples [5].

The observed dependence on $\mathrm{Cr}$ concentration confirms that the ASL excitation is in fact Cr-related. Unfortunately, a similar linear dependence on $\mathrm{Cr}$ concentration is expected for both discussed above the ASL excitation mechanisms. We have noticed, however, that these two mechanisms require distinctly different efficiency of $\mathrm{Cr}^{1+}+h_{V B}$ trapping process. This process should be relatively inefficient if the two-step ionization process dominates $\left(\mathrm{Cr}^{1+}\right.$ state must be metastably occupied for the efficient complementary ionization process) but must be efficient for the Auger-type transfer process. Otherwise, free electrons will not be generated in the $\mathrm{CB}$ in a sufficient concentration. The efficiency of trapping process can be changed by applying an external magnetic field. It is decreased at increased magnetic fields due to the spin selection rules. Once magnetic moments of free holes and spins of chromium ions become polarised, the $\mathrm{Cr}^{1+}(s=5 / 2)+\mathrm{h}_{\mathrm{VB}}$ $(j=3 / 2) \rightarrow \mathrm{Cr}^{2+}(s=2)$ process becomes a spin forbidden transition.

In the inset of Fig. 1 we show the ASL emission measured for two different magnetic fields ( $2.15 \mathrm{~T}$ and $5.95 \mathrm{~T}$ ) as compared to that measured without external magnetic field. The ASL emission clearly increases with an applied external magnetic field, which means that the two-step ionization mechanism is a dominant process and is responsible for the ASL excitation. One should be however cautious in making this statement general. The results of experiments shown in Fig. 2 can be interpreted in the way that different ASL excitation mechanisms may be important at different excitation intensities.

Concluding, the present results prove that the two-step ionization transitions of $\mathrm{Cr}$ ion result in appearance of the blue colour ASL DAP emission in chromium doped or contaminated ZnSe crystals.

\section{References}

[1] V.Yu. Ivanov, Yu.G. Semenov, M. Surma, M. Godlewski, Acta Phys. Pol. A 88, 743 (1995).

[2] V.Yu. Ivanov, Yu.G. Semenov, M. Surma, M. Godlewski, Phys. Rev. B, in press.

[3] M. Godlewski, W.E. Lamb, B.C. Cavenett, Solid Slate Commun. 39, 595 (1981).

[4] D.J. Robbins, P.J. Dean, P.E. Simmonds, H. Tews, in: Deep Centers in Semiconductors, Ed. S.T. Pantelides, Gordon and Breach Science Publ., 1992, p. 843.

[5] M. Surma, A.J. Zakrzewski, M. Godlewski, Phys. Rev. B 52, 11879 (1995). 